\title{
Anti-jamming techniques for multichannel SAR imaging
}

\author{
L. Rosenberg and D. Gray
}

\begin{abstract}
An airborne broadband jammer present in the mainbeam of a synthetic aperture radar (SAR) can potentially destroy a large region of the SAR image. In addition to this, multipath reflections from the ground, known as hot-clutter or terrain scattered interference will add a non-stationary interference component to the image. The goal of interference suppression for SAR is to successfully suppress these interferences while not significantly effecting the image quality by blurring, reducing the resolution or raising the sidelobe level. The paper provides an analysis of the degradation from hot-clutter, the limited restoration that multichannel imaging and slow-time space time adaptive processing (STAP) can provide and how fast-time STAP can improve the final image quality.
\end{abstract}

\section{Introduction}

Coherent SAR imaging is very sensitive to additive noise and an airborne broadband jammer has the potential to render it useless. Interference from the jammer can be modelled with two components; a direct-path signal and multipath reflections from the ground. The direct-path of the jammer signal is defined by a narrow azimuth region and while long integration times can be used to 'burnthrough' the interference [1], spatial degrees of freedom are required for effective cancellation. It has been shown that by combining the multichannel data from multiple pulses (slow-time) and performing slow-time STAP, much greater suppression is possible [2]. On the other hand, due to the diffuse reflection from the ground, the hot-clutter component is spread in azimuth and its properties can change rapidly with time, even over several adjacent pulses. This leads to a non-stationarity over slow-time and degrades the performance of slow-time adaptive filtering. Slow-time STAP works well for suppressing signals which are narrow in azimuth, though as the hot-clutter becomes more dominant, interference contributions spread in azimuth and become non-stationary over the coherent processing interval, resulting in images that are blurry and of poor quality. A secondary cause of non-stationarity comes from the changing motion between the SAR and jammer platforms which induces a bistatic Doppler shift for each scatterer. This effect is considered minimal in SAR as the jammer platform is typically a long distance away and the Doppler shift is relatively constant.

(C) The Institution of Engineering and Technology 2006

IEE Proceedings online no. 20045090

doi:10.1049/ip-rsn:20045090

Paper first received 30th September 2004 and in revised form 14th April 2005

The authors are with the Cooperative Research Center for Sensor, Signal and Information Processing (CSSIP) and Department of Electrical and Electronic Engineering, University of Adelaide, SA 5005, Australia

L. Rosenberg is also with the Defence, Science and Technology Organisation (DSTO)

E-mail: luker@eleceng.adelaide.edu.au
To effectively account for the effect of non-stationarity between pulses, cancellation of the interference should occur before azimuthal processing. Also the finite bandwidth of SAR means that multipath reflections are partially coherent with the direct-path jamming signal. This implies that temporal adaptive filtering is required within each pulse or over fast-time. Hot-clutter mitigation can therefore be undertaken by employing adaptive processing in both space and fast-time, forming a space/fast-time adaptive processor for each pulse [3, 4]. Ender [2] provides the most comprehensive study on SAR jamming, where a number of anti-jamming techniques were analysed and tested with simulation parameters mirroring the AER-II SAR. A number of spatial only methods were tested, such as the optimum anti-jamming filter using a known interference covariance, adaptive anti-jamming filters using estimates of the interference covariance and techniques using constraints. Ender then formulated a space/slow-time anti-jamming filter with image reconstruction using conditional expectation. Results indicated that slow-time STAP provides superior interference cancellation than spatial only filtering. It should be noted that hot-clutter was not included in any of this analysis.

Klemm [5] has also looked at anti-jamming for SAR by considering fast-time STAP suppression for the directpath only signal. A jammer and noise model specified by Compton [6] was applied to space/fast-time filtering to range compressed simulated data. Results showed that, firstly, fast-time filtering will degrade SAR resolution by broadening the point spread function (PSF) mainlobe in range and increasing its sidelobes and, secondly, as range resolution increased so did the sensitivity to filtering. Due to the filter weights changing over azimuth, these effects were worse when focussing near to the STAP filter notch. It was found that a larger number of elements in the array can keep the notches narrower and hence reduce the influence of jammer suppression.

It is unclear when hot-clutter was first identified as a problem in airborne radar, but mainstream publications on hot-clutter suppression techniques have been available since the mid-nineties, specifically at the Adaptive Sensor Array Processing (ASAP) conferences, 1995-1997. There 
are currently many different methods for suppressing hot-clutter (see [7, 8]), but only those involving fast-time sampling are relevant to this paper. Also, these publications are focussed primarily on airborne radar and the application of hot-clutter suppression for SAR is a problem that has not yet been addressed.

In this paper, the effect of hot-clutter on SAR imaging is demonstrated in Section 2 using models based on the work of Fante and Torres [4]. Section 3.1 describes a multichannel imaging algorithm to determine the effect of long integration times. Both optimal slow-time and fast-time STAP algorithms for SAR imaging are then described in Sections 3.2 and 3.3. Simulations results are presented in Section 4 and quantified in Section 5 to measure the effectiveness of interference rejection for these three algorithms. To simplify the analysis, it is assumed that the interference waveform is known, resulting in an idealised scenario where the training data waveform is identical to that of the jamming component of the received data vector. This approach is used to determine the potential of the proposed algorithms. However, a preliminary look at the effect of non-ideal training data is included to demonstrate the performance likely to be achieved in a real system.

\section{System models}

\subsection{SAR signal model}

Consider a SAR travelling along the $y$-axis, imaging a point in the slant-plane $x \in\left[X_{c}-X_{0}, X_{c}+X_{0}\right], y \in\left[-Y_{0}, Y_{0}\right]$. An $N$ channel linear antenna array with equi-spaced receivers is used in the azimuth direction to provide spatial degrees of freedom. For a real SAR system, the total ground return for the $n$th antenna is the integral over all scatterers with radar cross-section (RCS), $f(x, y)$

$$
g_{n}(t, u)=\int_{y} \int_{x} f(x, y) s_{n}(t, u, x, y) \mathrm{d} x \mathrm{~d} y
$$

Ignoring amplitudes, the form of the received signal model after range processing is given by

$$
s_{n}(t, u, x, y)=\exp \left[-j \omega_{c} \tau_{n}(u, x, y)\right] \operatorname{sinc}\left[B \pi\left(t-\tau_{n}(u, x, y)\right)\right]
$$

where the carrier frequency is $\omega_{c}(\mathrm{rad} / \mathrm{s})$ with bandwidth $B$ $(\mathrm{Hz})$ and the variables $(t, u)$ represent fast-time within a pulse and the SAR platform position respectively. If a signal is transmitted from the centre of the linear array when the SAR platform is at $(0, u)$, then the relative delay for the $n$th channel is independent of fast-time and is given by

$$
\tau_{n}(u, x, y)=\frac{1}{c}\left[R(x, y-u)+R\left(x, y-u-d_{n}\right)\right]
$$

where $R(x, y)=\sqrt{x^{2}+y^{2}}$ and the antenna spacing $d_{n}=n \delta$ with $n \in[-(N-1) / 2,(N-1) / 2]$ for $N$ (odd) antenna elements. In the fast-time frequency domain, $\omega \in\left[\omega_{c}-B \pi, \omega_{c}+B \pi\right]$ and the received signal model is given by

$$
s_{n}(\omega, u, x, y)=\exp \left[-j \omega \tau_{n}(u, x, y)\right]
$$

If the SAR is being jammed by an airborne platform, there will be two extra components required in the data model, the direct-path jammer $z_{n}^{d p}(\cdot)$ and the ground reflected path (hot-clutter) $z_{n}^{h c}(\cdot)$. These signals with the addition of receiver system noise $v(\cdot)$, form the components of the received SAR data waveform (if there were moving targets in the scene, these would be additional components)

$$
x_{n}(t, u)=g_{n}(t, u)+z_{n}^{d p}(t, u)+z_{n}^{h c}(t, u)+v_{n}(t, u)
$$

\subsection{Jammer signal model}

Consider the geometry of Fig. 1 where the ground is discretely sampled and the three points of interest have a given range, azimuth and velocity. Defining $x_{J, k}=\left|x_{J}-x_{k}\right|, \quad y_{J, k}=\left|y_{J}-y_{k}\right|$ and $y_{p, k}=\left|u-y_{k}\right|$, the radial distances $R_{p, k}=R\left(x_{k}, y_{p, k}\right)$ and $R_{J, k}=R\left(x_{J, k}, y_{J, k}\right)$ can be calculated using Pythagoras. The direct-path length can be calculated similarly by defining $y_{p, J}=\left|u-y_{J}\right|$ and using $R_{d, 0}=R\left(x_{J}, y_{p, J}\right)$. Unit vectors in each direction are indicated by $\tilde{\boldsymbol{R}}_{d, 0}, \tilde{\boldsymbol{R}}_{J, k}$ and $\tilde{\boldsymbol{R}}_{p, k}$.

The bistatic jammer model is adapted from [4] and assumes there are $K$ hot-clutter scatterers within the area on the ground that is being irradiated by the jammer. It is assumed that the jammer platform is directing its transmit beams to achieve maximum interference power on the SAR platform for both the direct-path and terrain scattered components. These components and the receiver noise are combined into a single variable, $z_{n}(\cdot)=z_{n}^{d p}(\cdot)+z_{n}^{h c}(\cdot)+$ $v_{n}(\cdot)$, for this formulation. If the time index used in the jammer waveform is written as the sum of fast- and slowtime, then the output of the $n$th receiver owing to the jammer $z_{n}(\cdot)$, is the superposition of the delayed reflections from each scatterer, i.e.

$$
z_{n}(t, u)=\sum_{k=0}^{K} b_{k} J\left(t+u / v_{p}-\tilde{\tau}_{n}\left(t, u, x_{k}, y_{k}\right)\right)+v_{n}(t, u)
$$

where the SAR platform speed is $v_{p}$ and $J(\cdot)$ is the signal at the jammer platform modelled as broadband Gaussian noise with zero mean and variance $\sigma_{J}^{2}$. The bistatic delay $\tilde{\tau}_{n}(\cdot)$ is simplified below and $b_{k}$ is defined as the relative magnitude between the direct jammer signal and the jammer signal reflected by the $k$ th scatterer. The zero index refers to the direct-path where $b_{0} \equiv 1$, while the $K$ hot-clutter scatterers have a magnitude relative to the direct-path given by

$$
b_{k}=\left(\frac{G_{r, k}}{G_{r, 0}} \frac{\sigma_{k} A_{k}}{4 \pi}\right)^{1 / 2} \frac{R_{d, 0}}{R_{p, k} R_{J, k}}, \quad k>0
$$

where $A_{k}$ is the effective area, $\sigma_{k}$ the bistatic RCS, $G_{r, 0}$ is the receive gain in the direction of the direct-path signal and $G_{r, k}$ the corresponding gain in the direction of the $k$ th scatterer. The noise signal $v_{n}(\cdot)$ represents the receiver noise for each channel. It is also modelled as white Gaussian noise with zero mean and variance $\sigma_{v}^{2}$. Equation

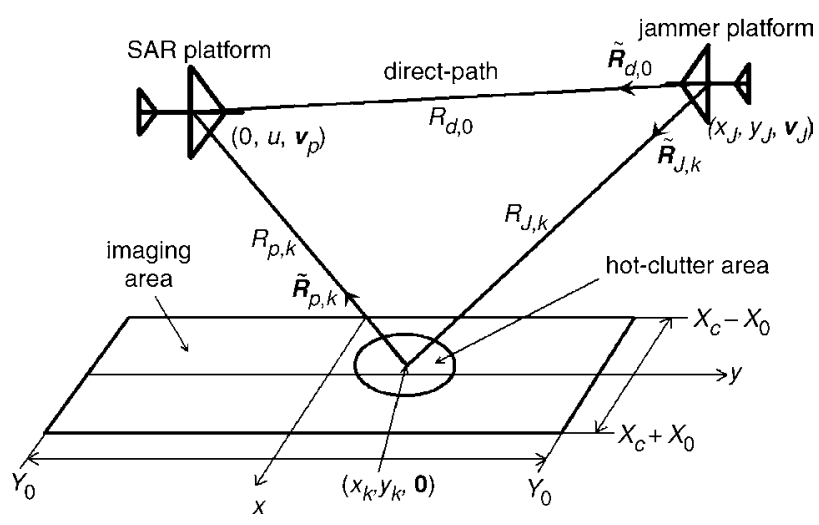

Fig. 1 Jammer geometry 
(6) can be simplified using the standard narrow-band assumption which implies that the jammer signal can be written as a separable function

$$
\begin{aligned}
z_{n}(t, u)= & \sum_{k=0}^{K} b_{k} J\left(t+u / v_{p}\right) \exp \left[-j \omega_{c} \tilde{\tau}_{n}\left(t, u, x_{k}, y_{k}\right)\right] \\
& +v_{n}(t, u)
\end{aligned}
$$

For further processing, it is useful to separate the slow- and fast-time components of the bistatic delay [4]. Consider the single channel radial distance due to a scatterer at $(x, y)$, when the SAR platform is sampled at fast-time $t_{l}$ corresponding to position, $u_{l}=v_{p} t_{l}$

$$
\begin{aligned}
R\left(x, y-u-u_{l}\right) & =\sqrt{x^{2}+\left(y-u-u_{l}\right)^{2}} \\
& =\sqrt{x^{2}+(y-u)^{2}-2(y-u) u_{l}+u_{l}^{2}} \\
& \simeq R(x, y-u)-\frac{2(y-u) u_{l}-u_{l}^{2}}{2 R(x, y-u)}
\end{aligned}
$$

where only a first order binomial expansion has been considered. This expression can then be separated in terms of slow and fast-time components

$$
R\left(x, y-u-u_{l}\right)=R(x, y-u)-\frac{(y-u) u_{l}}{R(x, y-u)}+\frac{u_{l}^{2}}{2 R(x, y-u)}
$$

where the third term is negligible when $\left|u_{l}\right| \ll|y-u|$. If the offset angle for the scatterer $(x, y)$ is defined as

$$
\theta(x, y)=\arctan \left(\frac{y}{x}\right)
$$

then the phase of the exponential can be written as

$$
\begin{aligned}
-\omega_{c} \tilde{\tau}_{n}(t, u, x, y) & =-\frac{\omega_{c}}{c}\left[R(x, y-u)-\sin \theta(x, y-u) v_{p} t_{l}\right] \\
& =-\omega_{c} \tilde{\tau}_{n}(u, x, y)-\omega_{d} t_{l}
\end{aligned}
$$

where

$$
\omega_{d}=-\frac{\omega_{c}}{c} \sin \theta(x, y-u) v_{p}
$$

is the fast-time Doppler shift. For a bistatic geometry, the delays and Doppler shifts are more complicated. Using the geometry in Fig. 1, the bistatic delays for the $k$ th scatterer are

$$
\tilde{\tau}_{n}\left(u, x_{k}, y_{k}\right)= \begin{cases}\frac{1}{c} R\left(x_{J}, y_{p, J}-d_{n}\right), & k=0 \\ \frac{1}{c}\left[R\left(x_{J, k}, y_{J, k}\right)+R\left(x_{k}, y_{p, k}-d_{n}\right)\right], & k>0\end{cases}
$$

with fast-time Doppler frequencies given by the inner product of the following vectors (in bold)

$$
\omega_{d, k}= \begin{cases}\frac{\omega_{c}}{c}\left(v_{p}-v_{J}\right)^{T} \tilde{\boldsymbol{R}}_{d, 0}, & k=0 \\ \frac{\omega_{c}}{c}\left(\boldsymbol{v}_{p}^{T} \tilde{\boldsymbol{R}}_{p, k}-\boldsymbol{v}_{J}^{T} \tilde{\boldsymbol{R}}_{J, k}\right), & k>0\end{cases}
$$

The jammer signal can then be written as

$$
\begin{aligned}
z_{n}(t, u)= & \sum_{k=0}^{K} b_{k} J(t) \exp \left[-j \omega_{c} \tilde{\tau}_{n}\left(u, x_{k}, y_{k}\right)\right] \exp \left[-j \omega_{d, k} t_{l}\right] \\
& +v_{n}(t, u)
\end{aligned}
$$

The hot-clutter area irradiated by the jammer contains $K$ scatterers and is modelled with a specular component at the centre of the hot-clutter area surrounded by diffuse components. Its RCS is modelled with a two-dimensional Gaussian function with independent $x$ and $y$ variables. Since the location of both platforms vary from pulse to pulse, the ground area irradiated by the jammer will also vary.

\subsection{Simulation models}

If these models are combined in a simulation, the effect of hot-clutter on a SAR image can be seen. Figure 2 shows a number of point scatterers and the synthetic SAR image which has been formed. For azimuth focussing, a multichannel imaging algorithm is used as described in Section 3.1. Details of the simulation and a summary of the parameters used are given in Section 4.

Figure $2 c$, with added hot-clutter, shows so much blurring that the original image is almost indecipherable. To quantify this degradation, a number of image metrics are presented in Section 5 and applied to the SAR images with varying hot-clutter power levels.

\section{Jammer suppression for SAR}

Three alternative algorithms are now presented which have the ability to both reject interferences and perform
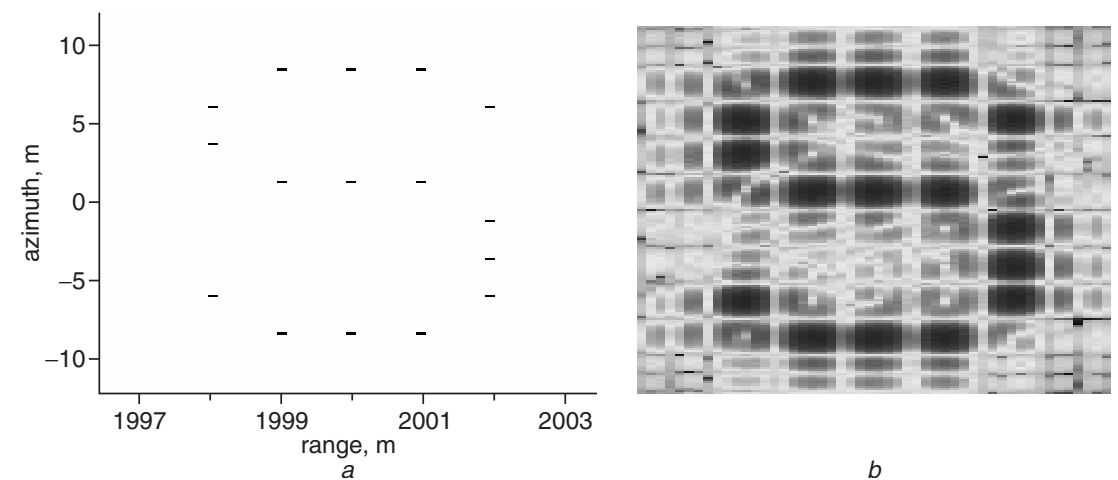

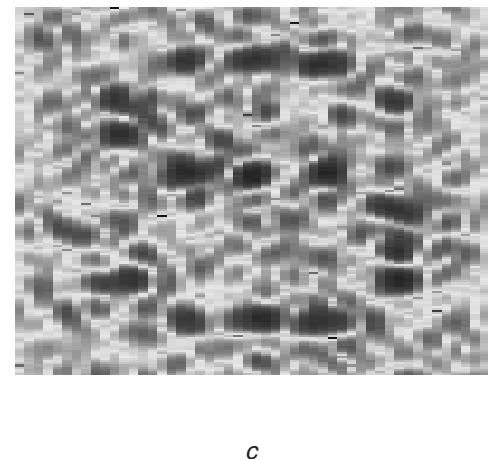

Fig. 2 Image comparison

$a$ Point scatterers

$b$ SAR image

$c$ SAR image with hot-clutter 
SAR imaging. The first is a space/slow-time multichannel imaging algorithm which relies on a large number of pulses to 'burn-through' the interference [1]. The second algorithm is optimal slow-time STAP which has been shown to produce good jammer suppression for signals that are stationary over the coherent processing interval and are narrow in azimuth [9]. The third algorithm is optimal fast-time STAP and is required for non-stationary interference [4], which changes between pulses and is incident in the SAR mainbeam. This algorithm is also able to account for any non-stationarity owing to changing geometry between pulses. Table 1 summarises these points.

\subsection{Multichannel SAR imaging}

High resolution SAR imaging requires a much longer integration time than conventional airborne radar. This is a desirable property for rejecting noise jammers as it enables the ground return to 'burn-through' the interference [1]. One suitable method for coherent integration/imaging of a multichannel SAR is spatial matched filter (MF) interpolation in the $\left(\omega, k_{u}\right)$ domain [10]. This algorithm uses a single frequency domain MF at a fixed range and performs range migration compensation by a Stolt interpolation to map from the measured to the image domain.

Multichannel imaging requires compensating for the phase difference of each antenna in the linear array (this is also known as beamforming). The reference signal $s_{n}(\cdot)$ is designed to match the data signal $x_{n}(\cdot)$ at the specified point $(x, y)$ and is given by (4). Both signals are stacked to give the signal vectors

$$
\begin{aligned}
\boldsymbol{s}(\omega, u, x, y) & =\left[s_{-(N-1) / 2}(\cdot), \ldots, s_{(N-1) / 2}(\cdot)\right]^{T} \\
\boldsymbol{x}(\omega, u) & =\left[x_{-(N-1) / 2}(\cdot), \ldots, x_{(N-1) / 2}(\cdot)\right]^{T}
\end{aligned}
$$

The imaging model in the $(\omega, u)$ domain, (1) can be generalised to

$$
\boldsymbol{x}(\omega, u)=\int_{y} \int_{x} f(x, y) \boldsymbol{s}(\omega, u, x, y) \mathrm{d} x \mathrm{~d} y
$$

where $f(x, y)$ is the reflectivity of the point being imaged. It was originally shown by Ender [11] and later extended for multiple range bins [10], that, with a slight extension to include multiple channels, the inverse of this equation is given by

$$
f(x, y)=\int_{k_{u}} \int_{\omega} \boldsymbol{s}^{H}\left(\omega, k_{u}, x, y\right) \boldsymbol{x}\left(\omega, k_{u}\right) \mathrm{d} \omega \mathrm{d} k_{u}
$$

In this equation, the beamforming has been accomplished by an inner product between the measured data and the reference signal. Implementing this algorithm requires a reference signal defined at the point $\left(X_{c}, 0\right)$ giving $s_{\mathrm{ref}, n}\left(\omega, k_{u}\right)=s_{n}\left(\omega, k_{u}, X_{c}, 0\right)$ and hence the inner product in the $\left(\omega, k_{u}\right)$ domain is given by

$$
F\left(\omega, k_{u}\right)=s_{\text {ref }}^{H}\left(\omega, k_{u}\right) x\left(\omega, k_{u}\right)
$$

To extend this algorithm to include slow-time vectors, (20) can be rewritten to explicitly perform a discrete convolution over $M$ pulses in the $u$ domain

$$
\begin{aligned}
F(\omega, u) & =\boldsymbol{s}_{\text {ref }}^{H}(\omega, u) * \boldsymbol{x}(\omega, u) \\
& =\sum_{m=1}^{M} \boldsymbol{s}_{\mathrm{ref}}^{H}\left(\omega, u-u_{m}\right) \boldsymbol{x}\left(\omega, u_{m}\right)
\end{aligned}
$$

This equation can be rewritten by forming an inner product over space/slow-time vectors stacked over the entire range of $u$. To maintain the phase centre at the centre of the synthetic array, the centre of the imaging patch occurs at $u_{M / 2}$ and hence both the slow-time steering and data vectors can be stacked over pulse delays where $u$ varies over the set $u_{1}, u_{2}, \ldots, u_{M}$,

$$
\begin{aligned}
S_{\mathrm{ref}}(\omega, u) & =\left[s_{\mathrm{ref}}\left(\omega, u-u_{1}\right), \ldots, \boldsymbol{s}_{\mathrm{ref}}\left(\omega, u-u_{M}\right)\right]^{T} \in \mathcal{C}^{M N \times 1} \\
\boldsymbol{X}(\omega) & =\left[\boldsymbol{x}\left(\omega, u_{1}\right), \ldots, \boldsymbol{x}\left(\omega, u_{M}\right)\right]^{T} \in \mathcal{C}^{M N \times 1}
\end{aligned}
$$

so that (21) can be written as

$$
F(\omega, u)=S_{\text {ref }}^{H}(\omega, u) X(\omega)
$$

To form the final image, the result must be Fourier transformed into the $\left(\omega, k_{u}\right)$ domain and then mapped from the measured to the image domain. While the sampled data is evenly spaced, once transformed into the $\left(k_{x}, k_{y}\right)$ spatial frequency domain, it becomes non-evenly spaced. To form an estimate of the reflectivity function $f(\cdot)$, range migration compensation is implemented using Stolt Interpolation with a sinc function smoothed with a Hamming window in the $\left(k_{x}, k_{y}\right)$ domain. A two-dimensional inverse Fourier transform then maps the spatial frequencies into $(x, y)$ image coordinates. A block diagram of this algorithm is presented in Fig. 3 where the wavenumber $k=\omega / c$. Note that the coherent averaging over pulses in the Fourier transform $u \rightarrow k_{u}$ can be interpreted as 'burn-through'.

\subsection{Slow-time STAP for stationary jammer suppression}

Principal causes of non-stationarity are due to the relative motion between the two platforms and the changing super-position of the direct-path and terrain scattered components of the interference. The degree of non-stationarity will depend on the relative power between these components in addition to the geometrical and physical features of the ground which vary from pulse to pulse. If the relative power of the direct-path signal is much greater then the terrain scattered component, the total interference can be classed as 'approximately stationary' and less intensive filtering using slow-time STAP algorithms may be sufficient to remove the predominant interference.

\section{Table 1: Jammer suppression techniques}

\begin{tabular}{ll}
\hline Algorithm & Properties \\
\hline No adaptation & Can suppress only with large number of pulses (burn-through) \\
Slow-time STAP & Shown to produce good jammer suppression for stationary interferences which are narrow in azimuth \\
Fast-time STAP & Accounts for changing geometry between pulses \\
& Necessary for non-stationary interference incident in the SAR mainbeam \\
\hline
\end{tabular}




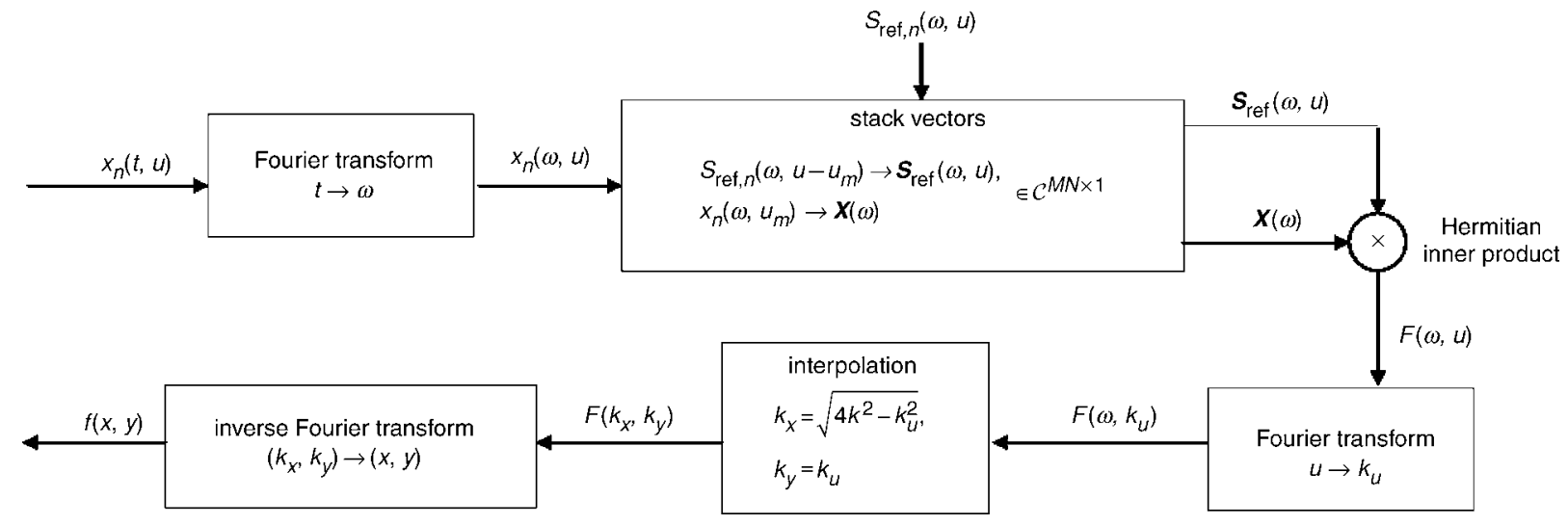

Fig. 3 Multichannel spatial/MF imaging

Optimal slow-time STAP involves replacing the slowtime reference or steering vector with the optimal weight for each frequency to maximise the signal to interference plus noise ratio (SINR) for each $(\omega, u)$

$$
\tilde{F}(\omega, u)=\boldsymbol{W}^{H}(\omega, u) \boldsymbol{X}(\omega)
$$

where

$$
\boldsymbol{W}(\omega, u)=\gamma \hat{\boldsymbol{R}}_{I}^{-1} \boldsymbol{S}_{\mathrm{ref}}(\omega, u) \in \mathcal{C}^{M N \times 1}
$$

The chosen optimisation criteria implies that an arbitrary scaling be used with this weight (e.g. $\gamma \equiv 1$ ) and therefore no constraints are imposed [5]. (The use of constraints to prevent signal suppression will be reported in future publications). The interference plus noise space-time covariance is estimated by averaging over $L$ frequency bins with $\mu \mathrm{dB}$ of diagonal loading

$$
\hat{\boldsymbol{R}}_{I}=\frac{1}{L} \sum_{l=1}^{L} \boldsymbol{Z}\left(\omega_{l}\right) \boldsymbol{Z}^{H}\left(\omega_{l}\right)+\mu \boldsymbol{I}_{M N} \in \mathcal{C}^{M N \times M N}
$$

where $\boldsymbol{Z}(\cdot)$ is the interference plus noise space/slow-time vector defined similarly to (22). It is assumed that techniques as described in [2 and 9] can be used to determine an appropriate $\boldsymbol{Z}(\cdot)$. The diagonal loading acts to minimise the norm of the weight vector, thereby reducing adaptive sidelobes, regularising the estimated covariance inverse when $L<N M$ and improving the overall performance of the adaption [13-15].

Since this paper is focussed on formulating a comparison between different anti-jamming techniques, an ideal training situation is considered where the actual interference plus noise waveform is used to form $\hat{\boldsymbol{R}}_{I}$. Future work will address more realistic training situations.

The final step in forming a SAR image is to perform range migration compensation by using Stolt interpolation and inverse Fourier transforming as described in the previous section. This process is described by the block diagram in Fig. 4.

\subsection{Fast-time STAP for hot-clutter suppression in $S A R$}

Interferences which are non-stationary require fast-time processing for effective cancellation. This formulation combines both spatial and fast-time samples to create a space/ fast-time processor which forms new weights for each pulse $u_{1}, u_{2}, \ldots, u_{M}$. Filtering SAR data in fast-time is equivalent to beamforming the spatial channels for each pulse. The form of the fast-time steering vector therefore contains the spatial delay multiplied by the time-varying component of (2)

$$
\overline{\boldsymbol{s}}_{\text {ref }, n}(t, u)=\exp \left[-j \omega_{c} \bar{\tau}_{n}(u)\right] \operatorname{sinc}[B \pi t]
$$

where

$$
\begin{aligned}
\bar{\tau}_{n}(u) & =\frac{1}{c}\left[R\left(X_{c}, u+d_{n}\right)-R\left(X_{c}, u\right)\right] \\
& \simeq-\frac{d_{n}}{c} \sin \left(\arctan \left(u / X_{c}\right)\right)
\end{aligned}
$$

This approximation can be made as the centre range is much greater than the imaging swath range, $X_{c} \gg X_{0}$. Fast-time filtering is accomplished by a convolution over range bins. If the fast-time steering vector and the data vector are first spatially stacked and then stacked over range bins $t_{1}, t_{2}, \ldots, t_{L}$

$$
\begin{aligned}
\overline{\boldsymbol{s}}_{\mathrm{ref}}\left(t-t_{l}, u\right)= & {\left[\bar{s}_{\mathrm{ref},-(N-1) / 2}\left(t-t_{l}, u\right), \ldots,\right.} \\
& \left.\bar{s}_{\mathrm{ref},(N-1) / 2}\left(t-t_{l}, u\right)\right]^{T} \in \mathcal{C}^{N \times 1} \\
\overline{\boldsymbol{S}}_{\mathrm{ref}}(t, u)= & {\left[\overline{\boldsymbol{s}}_{\mathrm{ref}}\left(t-t_{1}, u\right), \ldots, \overline{\boldsymbol{s}}_{\mathrm{ref}}\left(t-t_{L}, u\right)\right]^{T} \in \mathcal{C}^{L N \times 1} }
\end{aligned}
$$

and

$$
\begin{aligned}
\boldsymbol{x}\left(t_{l}, u\right) & =\left[x_{-(N-1) / 2}\left(t_{l}, u\right), \ldots, x_{(N-1) / 2}\left(t_{l}, u\right)\right]^{T} \in \mathcal{C}^{N \times 1} \\
\boldsymbol{X}(u) & =\left[\boldsymbol{x}\left(t_{1}, u\right), \ldots, \boldsymbol{x}\left(t_{L}, u\right)\right]^{T} \in \mathcal{C}^{L N \times 1}
\end{aligned}
$$

then the following equation can be written

$$
\begin{aligned}
\bar{x}(t, u) & =\sum_{l=1}^{L} \overline{\boldsymbol{s}}_{\mathrm{ref}}^{H}\left(t-t_{l}, u\right) \boldsymbol{x}\left(t_{l}, u\right) \\
& =\overline{\boldsymbol{S}}_{\mathrm{ref}}^{H}(t, u) X(u)
\end{aligned}
$$

Optimal fast-time STAP involves replacing the fast-time steering vector with the optimal weight for each pulse

$$
\bar{x}(t, u)=\overline{\boldsymbol{W}}^{H}(t, u) \boldsymbol{X}(u)
$$

where

$$
\overline{\boldsymbol{W}}(t, u)=\gamma \hat{\boldsymbol{R}}_{I}^{-1} \overline{\boldsymbol{S}}_{\mathrm{ref}}(t, u) \in \mathcal{C}^{L N \times 1}
$$

Once again, the maximum SINR criteria with $\gamma=1$ has been chosen to provide a comparison between the slowtime STAP formulation. The interference plus noise covariance is now determined by a sample matrix estimate over the previous $M^{\prime}$ pulses using the interference plus noise vector $\boldsymbol{Z}(u)$, defined similarly to (30). For example, on the 


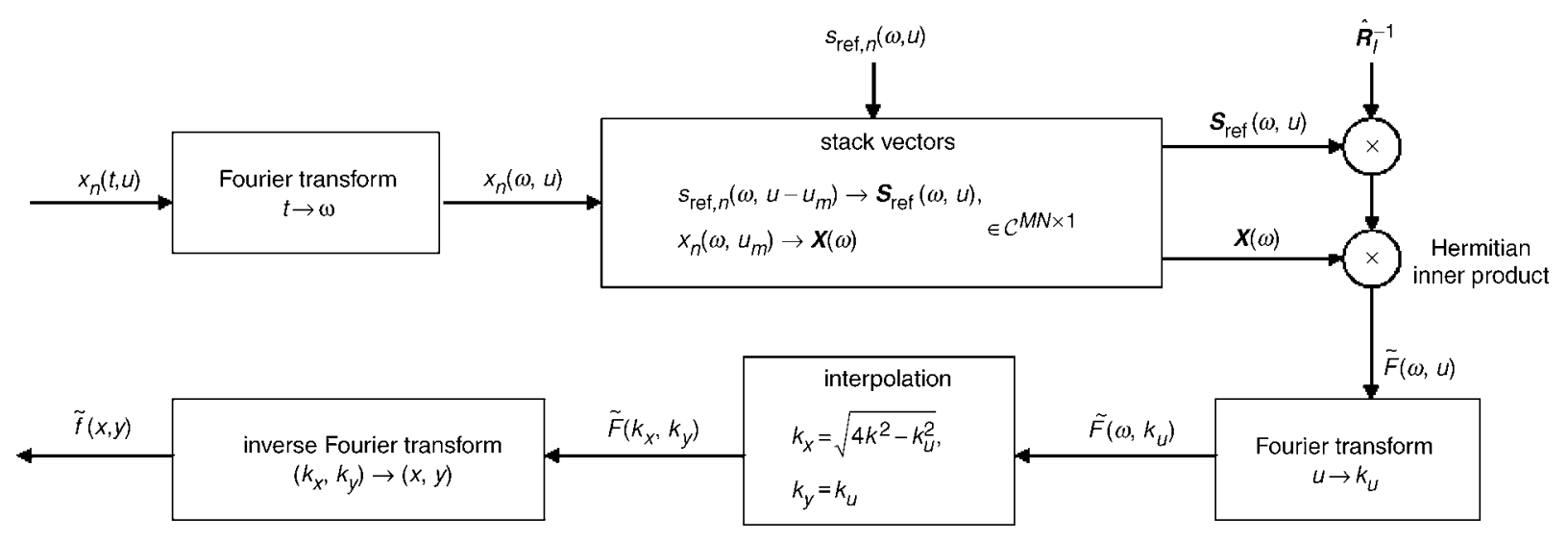

Fig. 4 Slow-time STAP with imaging

$m$ th pulse, $\Omega_{m} \equiv\left[m-M^{\prime}+1: m\right]$. The covariance estimate is again diagonally loaded by $\mu \mathrm{dB}$ to avoid a non-singular inverse

$$
\hat{\boldsymbol{R}}_{I}=\frac{1}{M^{\prime}} \sum_{\Omega_{m}} \boldsymbol{Z}\left(u_{m}\right) \boldsymbol{Z}^{H}\left(u_{m}\right)+\mu \boldsymbol{I}_{L N} \in \mathcal{C}^{L N \times L N}
$$

The modified single channel data $\bar{x}(\cdot)$ must then be focussed in azimuth. This is done using the imaging algorithm from Section 3.1. Figure 5 represents an overview of this algorithm.

\section{Simulated results}

An X-band simulation with $300 \mathrm{MHz}$ bandwidth and five spatial channels $(N=5)$ is used to compare these three algorithms with a varying ratio of hot-clutter power to direct-path interference power. The spatial channels are arranged in a linear array with half-wavelength spacing and are modelled as dipoles. The scenarios were chosen with the hot-clutter scatterers tightly constrained in angle with similar bistatic time delays. This implies that when the interference power is much greater than both the thermal noise and loading factor, the dominant component of the interference plus noise covariance will be of low rank. To demonstrate good suppression for fast-time STAP, the estimated interference covariance does not need averaging $\left(M^{\prime}=1\right)$ and a single realisation of the interference plus noise waveform can be used to form the covariance estimate. A derivation to justify this is included in the Appendix.

Both a PSF and sample image with target power $\sigma_{t}^{2}$, are simulated to test the different comparison metrics described in the following Section. Due to the different parameters given in Table 2, the clutter (or target) to interference plus noise ratios (CINR) are different for each scenario. If each of the hot-clutter scatterers are set to the same relative power, $b_{k}=b$ for $k=1 \ldots K$, then a range of hot-clutter powers relative to the direct-path interference power, can used to measure the effectiveness of the three different

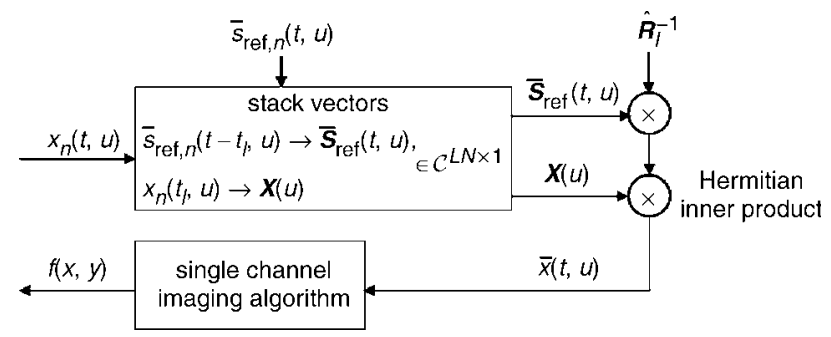

Fig. 5 Fast-time STAP
Table 2: Simulation parameters

\begin{tabular}{ll}
\hline Parameters & Value \\
\hline Carrier frequency $\left(f_{c}\right) /$ bandwidth $(B)$ & $10 / 0.3 \mathrm{GHz}$ \\
Number of elements $(N) /$ spacing $(\delta)$ & $5 /(\lambda / 2) \mathrm{m}$ \\
Platform speed $\left(v_{p}\right) /$ jammer speed $\left(v_{J}\right)$ & $200 / 100 \mathrm{~ms}^{-1}$ \\
Range resolution $\left(\Delta_{x}\right) /$ range centre $\left(X_{c}\right)$ & $1 \mathrm{~m} / 2000 \mathrm{~m}$ \\
Number of hot-clutter scatterers $(K)$ & 400 \\
Diagonal loading factor $(\mu)$ & $0 \mathrm{~dB}$ \\
Clutter (target) power $\left(\sigma_{t}^{2}\right) /$ noise power $\left(\sigma_{v}^{2}\right)$ & $20 \mathrm{~dB} / 0 \mathrm{~dB}$ \\
\hline SAR imaging area - point target: & \\
Direct-path power $\left(\sigma_{J}^{2}\right)$ & $40 \mathrm{~dB}$ \\
PRF ( $\left.f_{P R F}\right) /$ azimuth resolution $\left(\Delta_{y}\right)$ & $1.3 \mathrm{kHz} / 4 \mathrm{~m}$ \\
Azimuth swath size $\left(2 Y_{0}\right) /$ range swath size $\left(2 X_{0}\right)$ & $12 \mathrm{~m} / 4 \mathrm{~m}$ \\
Number of pulses $(M) /$ range bins $(L)$ & $100 / 58$ \\
\hline SAR imaging area - sample image: & \\
Direct-path power $\left(\sigma_{J}^{2}\right)$ & $80 \mathrm{~dB}$ \\
PRF ( $\left.f_{P R F}\right) /$ azimuth resolution $\left(\Delta_{y}\right)$ & $1.44 \mathrm{kHz} / 2.4 \mathrm{~m}$ \\
Azimuth swath size $\left(2 Y_{0}\right) /$ range swath size $\left(2 X_{0}\right)$ & $24 \mathrm{~m} / 10 \mathrm{~m}$ \\
Number of pulses $(M) /$ range bins $(L)$ & $180 / 102$ \\
\hline
\end{tabular}

algorithms. The CINR can be defined as

$$
C I N R=\frac{\sigma_{t}^{2}}{\sigma_{n}^{2}+\sigma_{J}^{2}\left(1+K^{2} b\right)}
$$

where it is assumed that all hot-clutter scatterers are correlated. Examples of the algorithm results are shown by the sample images in Fig. 6 and the PSFs in Fig. 7. From a visual inspection of both Figures, it appears that conventional multichannel imaging is completely degraded by the interference. The slow-time STAP algorithm has rejected the interference quite well when the hot-clutter power is weak, but the results indicate an increasing level of blurriness as the power increases. The fast-time STAP images manage to both suppress the interference and maintain image quality for all hot-clutter power levels. This is confirmed by the point spread functions, where the fasttime STAP has much lower sidelobes than both the other algorithms. Using the parameters from Table 1, the corresponding CINRs for the sample image are -82 and $-132 \mathrm{~dB}$.

With regard to the loading factor $\mu$, it has been shown that choosing approximately three times the sensor noise yields optimum results for most cases [14]. In this work however, the chosen loading factor is much less due to 

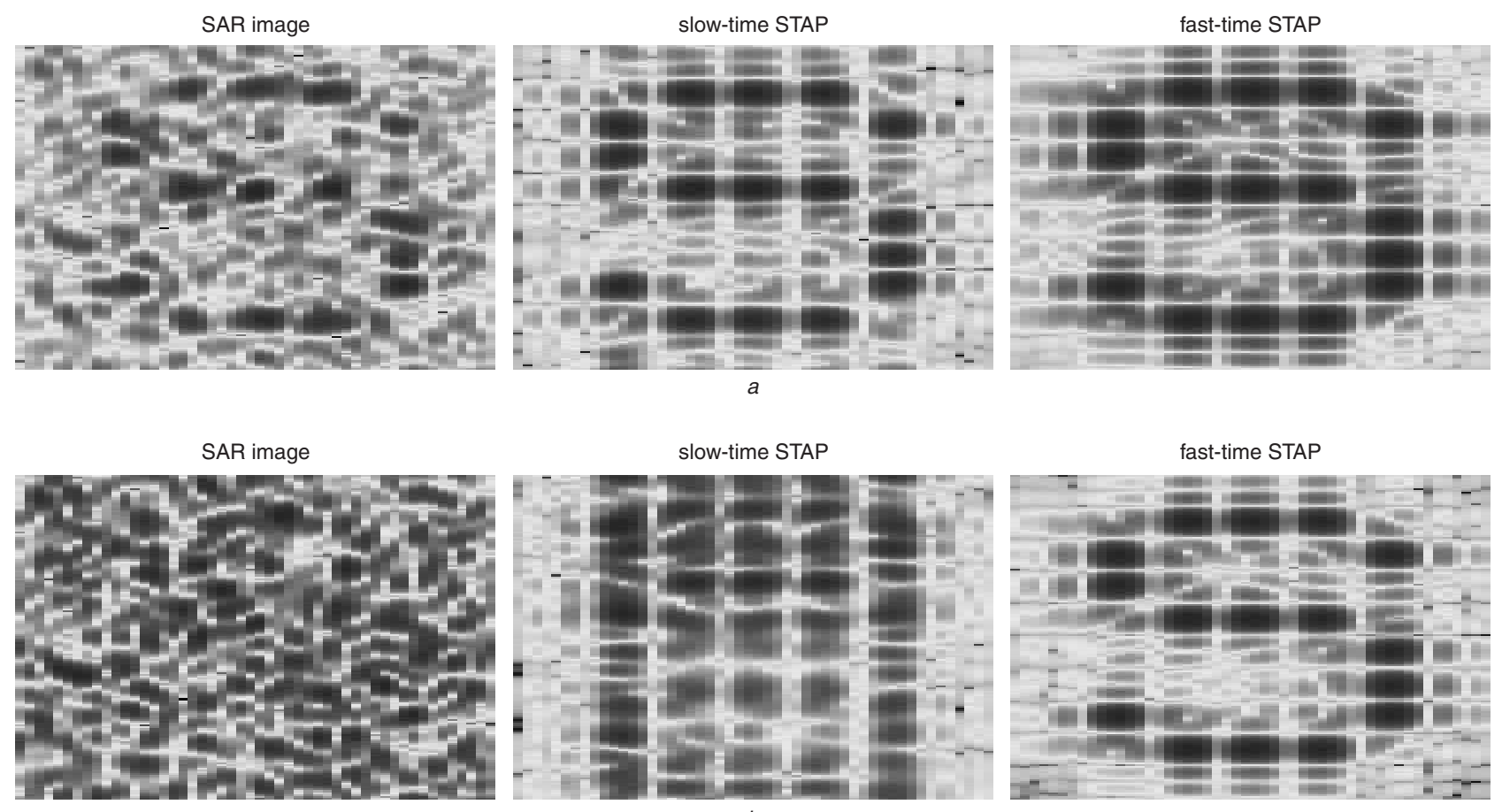

Fig. 6 SAR images

$a$ For $b=-30 \mathrm{~dB}$

$b$ For $b=20 \mathrm{~dB}$

the sample matrix estimate using a known interference waveform and the loading factor is used primarily to regularise the matrix for inversion. Other recent techniques for determining the loading factor are given in $[15,16]$.

\section{Image comparisons}

To obtain a quantitative measure of image degradation, the SINR and mean square error (MSE) can be calculated. Let $Y\left(x_{p}, y_{q}\right)$ denote either the conventional, slow-time or fasttime STAP images described above in the presence of signal, jammer and noise for image pixels $p=1 \ldots P$, $q=1 \ldots Q$. Correspondingly, $D\left(x_{p}, y_{q}\right)$ denotes the image in the presence of just the signal. The SINR is then defined as the ratio of the desired signal power to interference power:

$$
\operatorname{SINR}=\frac{\sum_{p, q}\left|D\left(x_{p}, y_{q}\right)\right|^{2}}{\sum_{p, q}\left|Y\left(x_{p}, y_{q}\right)-D\left(x_{p}, y_{q}\right)\right|^{2}}
$$

while the MSE is determined by the difference between the total desired signals:

$$
M S E=\frac{1}{P Q} \sum_{p, q}\left|Y\left(x_{p}, y_{q}\right)-D\left(x_{p}, y_{q}\right)\right|^{2}
$$

To provide a greater degree of accuracy, each of these metrics will be averaged over five independent realisations. Figure 8 presents the averaged values of SINR and MSE when the relative power of the hot-clutter interference is varied.

As the relative hot-clutter power level is increased for the three cases in Fig. 8, the SINR decreases and the MSE increases accordingly. While the slow-time STAP offers an improvement, the best suppression is achieved by fasttime STAP, which maintains good performance independent of the strength of the hot-clutter. Note that due to the sample matrix estimate being used for the slow-time STAP algorithm, its results will not converge to those of the fast-time STAP for small hot-clutter powers.
To further quantify the effect of the interference, the PSF is formed from a single scatterer at the centre of the imaging patch, such as in Fig. 7. Range and azimuth slices can be used to calculate the peak sidelobe ratio (PSR) and the integrated sidelobe ratio (ISLR). These are defined to be the difference between the mainlobe and greatest sidelobe and the ratio of all energy in the sidelobes to the energy in the mainlobe. Fig. 9 shows a comparison for the azimuthal slice of the ISLR and PSR for a single point scatterer as the relative hot-clutter power is varied. As the range PSR and ISLR remain almost constant for each of the three cases, only the azimuth ratios are analysed.

As expected when the interference level increases, the ISLR increases while the PSR decreases. Slow-time STAP appears to perform much better than the results from Fig. 8 owing to the different parameters chosen. Eventually it does degrade around $40 \mathrm{~dB}$ and is outperformed by fast-time STAP.

\section{Realistic training}

Realistic training is achieved by using one set of realisations for the jammer signal to estimate the weight vector and another independent set to contaminate the image to be processed. This is consistent with the training methods described in [9] where the interference-only signal can be measured at the end of a PRI where ground returns are very weak. Slow-time STAP does not change in performance with realistic training, since it still requires averaging over a number of pulses.

However, with fast-time STAP, there the two side-effects as described in [17]. The first is 'training modulation', which arises owing to averaging over a finite number of different realisations. The second is 'coherence modulation', which results from non-stationarity in the covariance estimate. It is important to understand the effect of realistic training data on this algorithm and how it effects a focussed SAR image.

To isolate the modulations, a simulation with only the direct-path signal is used as it represents a spatially 

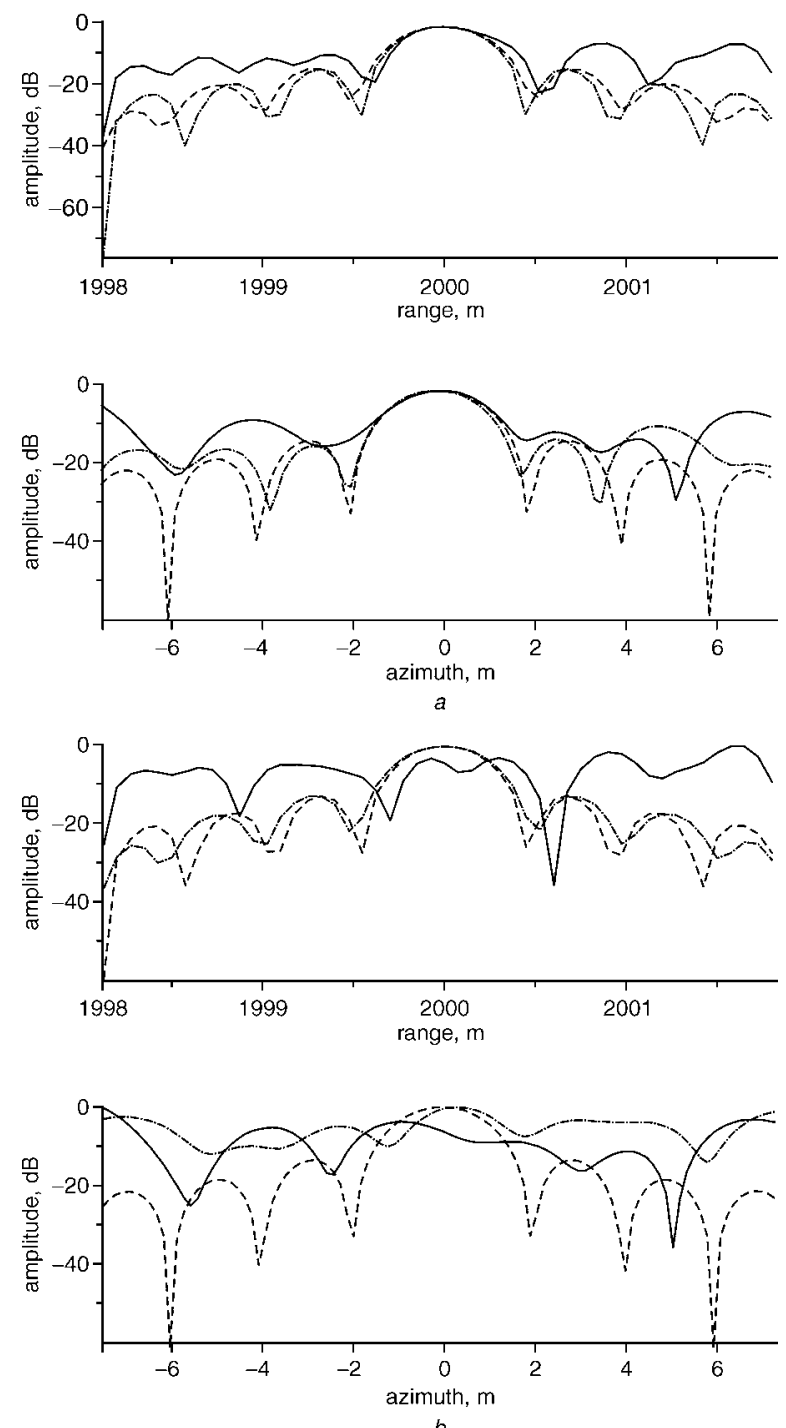

Fig. 7 SAR point spread function

$\begin{array}{ll}-.+. & \text { conventional imaging } \\ -\cdot-\cdot . & \text { slow-time STAP }\end{array}$

--- fast-time STAP

$a$ For $b=-10 \mathrm{~dB}$

$b$ For $b=60 \mathrm{~dB}$
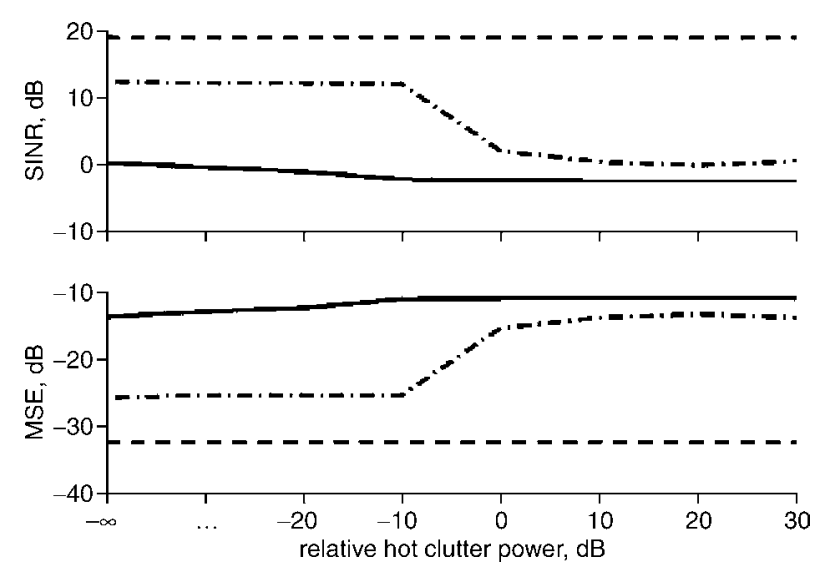

Fig. 8 SINR and MSE comparisons

conventional imaging

slow-time STAP

fast-time STAP

stationary environment. Unlike the ideal conditions in this study, using no averaging in a real system would give a very poor estimate of the interference covariance. As a result, there is a trade-off between the accuracy of the
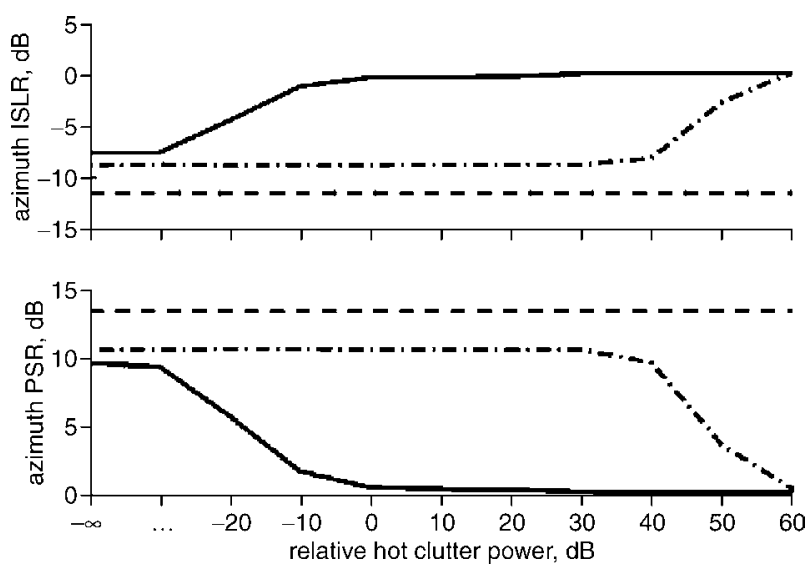

Fig. 9 ISLR and PSR comparisons

- conventional imaging slow-time STAP fast-time STAP

estimate and the modulation owing to training. Fig. 10 shows a PSF with no averaging $\left(M^{\prime}=1\right)$ and with significantly more averaging $\left(M^{\prime}=39\right)$.

Averaging over a greater number of pulses has the effect of raising the sidelobes of the PSF and skewing the range slice to the left. Although the mainlobes for the range slice are very similar, distortions in the sidelobes are approximately $5-10 \mathrm{~dB}$. This is much worse for the azimuth slice indicating that training modulation adds a significant distortion to the SAR image.

Similarly to isolate the coherency modulation, a simulation with no averaging $\left(M^{\prime}=1\right)$ is performed using realistic training data. Fig. 11 shows the PSF with 30, 40 and $50 \mathrm{~dB}$ of interference power.

As the interference power is increased, the fast-time STAP algorithm needs to adapt more strongly. This has the side effect of modulating the phase of the SAR signal and consequently the three images in Fig. 11 demonstrate raised sidelobe levels and increased blurring. Although there is some shape for the first two cases, when $b_{0}=50 \mathrm{~dB}$, imaging fails completely.

\section{Conclusion}

This paper has demonstrated how hot-clutter can degrade a multichannel SAR image and cause it to be completely
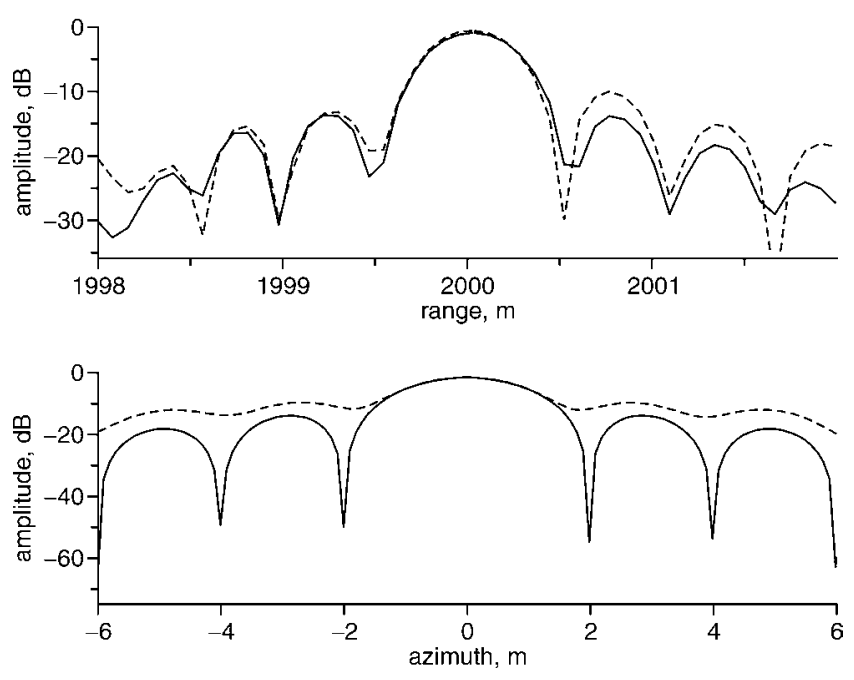

Fig. 10 PSF demonstrating training modulation

for $M^{\prime}=1$

for $M^{\prime}=39$ 

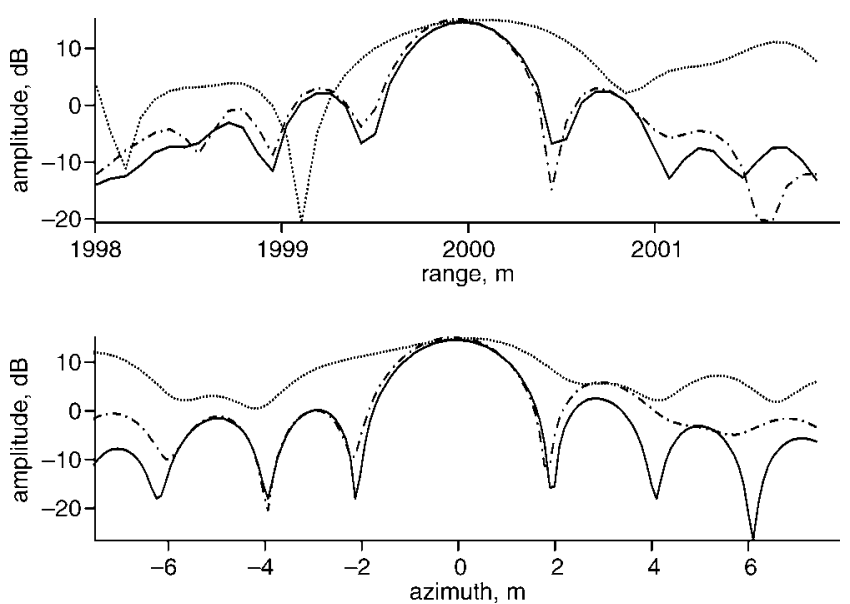

Fig. 11 PSF demonstrating coherency modulation

$\begin{array}{ll} & \text { for } b_{0}=30 \mathrm{~dB} \\ -\cdot-\cdot- & \text { for } b_{0}=40 \mathrm{~dB} \\ ---- & \text { for } b_{0}=50 \mathrm{~dB}\end{array}$

distorted. Three algorithms have been presented to show how both stationary and non-stationary interferences can be suppressed. The first algorithm was a space-time multichannel imaging algorithm and by using a large number of pulses, offers some interference reduction through the process of burn-through.

The second algorithm was optimal slow-time STAP, which has the ability to reject the direct-path interference and a small amount of hot-clutter. This property allows slow-time STAP to be able to use realistic training data without any compromise in performance.

The third algorithm, fast-time STAP has shown to be far more effective than the first two algorithms in the presence of strong hot-clutter. The drawback of this algorithm comes when using realistic training data. Being able to maintain phase coherency while suppressing the interference is hard to achieve and is the focus of future work.

\section{References}

1 Skolnik, M.: 'Radar handbook' (McGraw-Hill, 1990, 2nd edn.), pp. $1.9,9.17$

2 Ender, J.: 'Anti-jamming adaptive filtering for SAR imaging'. DGON IRS'98, Munich, 1998

3 Griffiths, L.J., Techau, P.M., Bergin, J.S., and Bell, K.L.: 'Space-time adaptive processing in airborne radar systems'. IEEE 2000 Int. Radar Conf., 2000

4 Fante, R.L., and Torres, J.A.: 'Cancellation of diffuse jammer multipath by an airborne adaptive radar', IEEE Trans. Aerosp. Electron. Syst., 1995, 31, (2), pp. 805-820

5 Klemm, R.: 'Principles of space-time adaptive processing' (The Institution of Electrical Engineers, 2002)

6 Compton, R.T. Jr.: 'The bandwidth performance of a two-element adaptive array with tapped delay-line processing', IEEE Trans. Antennas Propag., 1988, 36, pp. 5-14

7 Gabel, R.A., Kogon, S.M., and Rabideau, D.J.: 'Algorithms for mitigating terrain-scattered interference', Electron. Commun. Eng. J., 1999, pp. 49-56

8 Guerci, J.R., Goldstein, J.S., and Reed, I.S.: 'Optimal and adaptive reduced-rank STAP', IEEE Trans. Aerosp. Electron. Syst., 2000, 36, pp. $647-663$

9 Ward, J.: 'Space-time adaptive processing for airborne radar'. Technical report 1015, Lincoln Labs. MIT, 1994

10 Rosenberg, L., and Gray, D.: 'Multichannel SAR imaging using wavefront reconstruction'. Int. Radar Symp. Proc., 2004, pp. 155-160

11 Ender, J.H.G.: 'Space-time adaptive processing for synthetic aperture radar'. IEE Colloquium on Space-Time Adaptive Processing, 1998

12 Carlson, B.D.: 'Covariance matrix estimation errors and diagonal loading in adaptive arrays', IEEE Trans. Aerosp. Electron. Syst., 1998, 24, (4), pp. 397-401

13 Gierull, C.H.: 'Analysis of the small sample size performance of fast fully adaptive STAP techniques for MTI radar'. Technical Report
DRDC TR 2001-079, Defence Research \& Development Canada, Ottawa, Canada, October 2001

14 Cheremisin, O.P.: 'Efficiency of adaptive algorithms with regularized sample covariance matrix', Radiotekh. Elektron., 1982, 2, (10), pp. 1933-1941

15 Li, J., Stoica, P., and Wang, Z.: 'On robust capon beamforming and diagonal loading', IEEE Trans. Signal Process., 2003, 51, (7) pp. $1702-1715$

16 Ma, N., and Goh, T.: 'Efficient method to determine diagonal loading value'. Int. Conf. on Acoustics, Speech, and Signal Process., 2003

17 Rabideau, D.: 'Clutter and jammer multipath cancellation in airborne adaptive radar', IEEE Trans. Aerosp. Electron. Syst., 2000, 36, (2), pp. $565-583$

18 Hung, E.K., and Turner, R.M.: 'A fast beamforming algorithm for large arrays', IEEE Trans. Aerosp. Electron. Syst., 1983, AES-19, (4), pp. 589-607

19 Gierull, C.H.: 'Performance analysis of fast projections of the Hung-Turner type for adaptive beamforming', Signal Process. 1996, 50, (1-2), pp. 17-29

\section{Appendix: SMl estimate using ideal training data}

Consider the following rank one covariance estimate $\left(M^{\prime}=1\right)$ of the $m$ th pulse using the $L N \times 1$ interference plus noise signal $\boldsymbol{Z}(u)$, defined in Section 3.3

$$
\hat{\boldsymbol{R}}_{I}=\boldsymbol{Z}\left(u_{m}\right) \mathbf{Z}^{H}\left(u_{m}\right)+\mu \boldsymbol{I}_{L N} \in \mathcal{C}^{L N \times L N}
$$

where the covariance matrix has also been diagonally loaded to avoid a non-singular inverse. Using this covariance will perfectly cancel the interference if there is no averaging done and the CINR tends to infinity. Consider the following derivation, with the desired target represented by $\boldsymbol{T}$ and the dependence on $u_{m}$ has been dropped for $\boldsymbol{Z}\left(u_{m}\right)$

$$
\begin{aligned}
\bar{x}(t, u) & =\left[\left(\mathbf{Z Z}^{H}+\mu \boldsymbol{I}\right)^{-1} \boldsymbol{s}\right]^{H}(\boldsymbol{T}+\boldsymbol{Z}) \\
& =\boldsymbol{s}^{H}\left(\mathbf{Z Z}^{H}+\mu \boldsymbol{I}\right)^{-1}(\boldsymbol{T}+\boldsymbol{Z})
\end{aligned}
$$

Using the matrix inversion lemma gives:

$$
\begin{aligned}
\bar{x}(t, u)= & \boldsymbol{s}^{H}\left(\mu^{-1} \boldsymbol{I}-\mu^{-2} \boldsymbol{Z}\left(1+\mu^{-1} \boldsymbol{Z}^{H} \boldsymbol{Z}\right)^{-1} \boldsymbol{Z}^{H}\right)(\boldsymbol{T}+\boldsymbol{Z}) \\
= & \mu^{-1} \boldsymbol{s}^{H} \boldsymbol{T}+\boldsymbol{s}^{H}\left[\mu^{-1} \boldsymbol{Z}-\frac{\mu^{-2} \boldsymbol{Z} \boldsymbol{Z}^{H}(\boldsymbol{T}+\boldsymbol{Z})}{1+\mu^{-1} \boldsymbol{Z}^{H} \boldsymbol{Z}}\right] \\
= & \mu^{-1} \boldsymbol{s}^{H} \boldsymbol{T} \\
& +\boldsymbol{s}^{H}\left[\frac{\mu^{-1} \boldsymbol{Z}\left(1+\mu^{-1} \boldsymbol{Z}^{H} \boldsymbol{Z}\right)-\mu^{-2} \boldsymbol{Z} \boldsymbol{Z}^{H}(\boldsymbol{T}+\boldsymbol{Z})}{1+\mu^{-1} \boldsymbol{Z}^{H} \boldsymbol{Z}}\right] \\
= & \mu^{-1} \boldsymbol{s}^{H} \boldsymbol{T} \\
& +\boldsymbol{s}^{H}\left[\frac{\boldsymbol{Z}+\mu^{-1} \boldsymbol{Z} \boldsymbol{Z}^{H} \boldsymbol{Z}-\mu^{-1} \boldsymbol{Z} \boldsymbol{Z}^{H} \boldsymbol{T}-\mu^{-1} \boldsymbol{Z} \boldsymbol{Z}^{H} \boldsymbol{Z}}{\mu+\boldsymbol{Z}^{H} \boldsymbol{Z}}\right] \\
= & \mu^{-1} \boldsymbol{s}^{H} \boldsymbol{T}+\boldsymbol{s}^{H}\left[\frac{\boldsymbol{Z}-\mu^{-1} \boldsymbol{Z} \boldsymbol{Z}^{H} \boldsymbol{T}}{\mu+\boldsymbol{Z}^{H} \boldsymbol{Z}}\right]
\end{aligned}
$$

If the diagonal loading is small compared to the interference power, this can be reduced to

$$
\begin{aligned}
\bar{x}(t, u) & \simeq \mu^{-1} \boldsymbol{s}^{H} \boldsymbol{T}-\frac{\mu^{-1} \boldsymbol{s}^{H} \boldsymbol{Z} \boldsymbol{Z}^{H} \boldsymbol{T}}{\boldsymbol{Z}^{H} \boldsymbol{Z}} \\
& =\mu^{-1} \boldsymbol{s}^{H}\left[\boldsymbol{I}-\frac{\boldsymbol{Z} \boldsymbol{Z}^{H}}{\boldsymbol{Z}^{H} \boldsymbol{Z}}\right] \boldsymbol{T} \\
& =\mu^{-1} \boldsymbol{s}^{H} \boldsymbol{P}_{I} \boldsymbol{T}
\end{aligned}
$$

where $\boldsymbol{P}_{I}$ is a projection onto the space orthogonal to the interference. This result is commonly known as the HungTurner projection $[18,19]$. 